\title{
An aggregation function to solve multicriteria ranking problem involving several decision makers
}

\author{
Zoïnabo Savadogo ${ }^{1}$, Ruffin-Benoît M. Ngoie ${ }^{2 *}$, Berthold Ulungu E.-L. ${ }^{3,4}$, Blaise Somé ${ }^{1}$ \\ ${ }^{1}$ University of Ouagadougou, UFR-SEA/LANIBIO, Burkina Faso \\ ${ }^{2}$ ISP/Mbanza-Ngungu, Democratic Republic of Congo \\ ${ }^{3}$ ISTA/Kinshasa, Democratic Republic of Congo \\ ${ }^{4}$ Laboratory, MATHRO,University of Mons, Belgium \\ *Corresponding author E-mail: benoitmpoy@hotmail.com
}

Copyright (C) 2014 Zö̈nabo Savadogo et al. This is an open access article distributed under the Creative Commons Attribution License, which permits unrestricted use, distribution, and reproduction in any medium, provided the original work is properly cited.

\begin{abstract}
Multiple Criteria Decision Aiding (MCDA) has been studied in a single decision maker framework for a long time. Nowadays, the need to take into account several conflicting opinions handled by several decisions makers arises. So, researchers are interested with multicriteria problems involving several decision makers. In this context, to solve ranking problem, we develop an aggregation model of several additive value functions. Comparisons with a derivative ELECTRE I method is done on numerical data. Clearly, it appears that the proposed aggregation function is better according to calculation complexity and computation time. Way for further research in this field is proposed.
\end{abstract}

Keywords: Aggregation Function, Group Decision, Multicriteria Decision Aiding, Ranking Problem.

\section{Introduction}

Deciding is a very complex and difficult task. Some people argue that our ability to make decisions in complex situations is the main feature that distinguishes us from animals [1]. Till nineties, when a manager should take a decision, he could consult experts, focus on similar cases or use classical operations research techniques. Indeed, these techniques are essentially concerned to optimize a so-called economic function $[1,2]$. Since last three passed decades, experts focus on multicriteria paradigm which consists to guideline decision by several opposite objectives. Making decision nowadays goes beyond the strict framework of the traditional decision maker who isolates himself to make a decision [3]. The current trend involves a group of people concerned by the decision expertise. One must take into account several different points of view related to various experts or decision makers (DM) to reach a consensual view. Choice between alternatives is reached through a scalarizing process that aims to aggregate individual preferences into a collective preference. According to Jean-Luc Marichal [4], aggregation functions are generally defined and used to combine and summarize several numerical values into one so that the final result of the aggregation takes into account, in a prescribed manner, all individual values.

This paper aims at presenting an aggregation model of several additive value functions to solve a ranking problem with several DM. The multiplicity of decision makers highlights collective decision-making importance within organizations [5]. Indeed, each decision maker brings her or his judgment with respect to each action. Then, one obtains a collective ranking from which is computed a consensual result as indicated in group decision process (see Fig. 1 from [5]).

This paper is organized as follows: Section 2 presents multicriteria ranking problem with several DM, Section 3 is devoted to methods for solving ranking problem in group decision process, some concluding remarks are given in Section 4. 


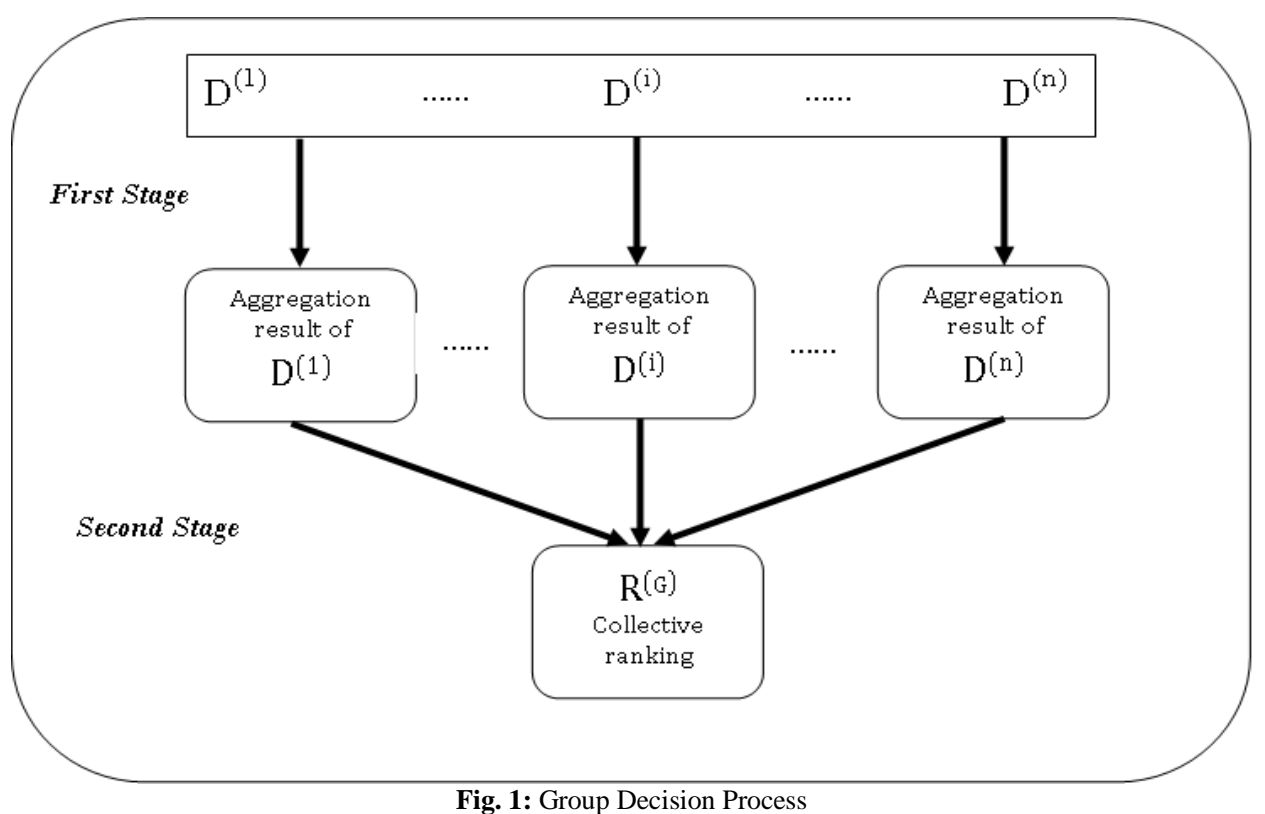

\section{Multicriteria ranking problem with multiple decision-makers (DM)}

\subsection{Multicriteria with multiple DM}

Most decision-aid problems involve multiple criteria as well as DMs. Such a problem arises when:

- We have 5 sets :

- $\quad \mathrm{D}=\left\{d_{1}, d_{2}, \ldots, d_{s}\right\}$ identifying all the $s$ DMs;

- $\quad \mathrm{A}=\left\{a_{1}, a_{2}, \ldots, a_{n}\right\}$ collecting the $n$ alternatives or actions;

- $\quad \mathrm{C}=\left\{g_{1}, g_{2}, \ldots, g_{m}\right\}$ determining the $m$ criteria;

- $\quad \mathrm{X}=\left\{x_{i j}=g_{j}\left(a_{i}\right), i=1, \ldots, n ; j=1, \ldots, m\right\}$ defining the performance ratings of alternative $i$ on criterion $j$;

- $\quad \mathrm{X}^{1}=\left\{x_{i j}^{l}=g_{j}\left(a_{i}\right), i=1, \ldots, \mathrm{n} ; j=1, \ldots, m ; l=1, \ldots, s\right\}$ defining the performance ratings of alternative $i$ on criterion $\mathrm{j}$ for $l^{\text {th }} \mathrm{DM}$.

- $\quad$ No DM is dictatorial one ;

- No alternative dominates all other ones on each criterion ;

- We need a multicriteria decision aid to found a good compromise.

\subsection{Ranking problem}

This problematic, denoted $\mathrm{P}_{\gamma}$ in literature, consists to completely or partially classify alternatives $a_{i} \in \mathrm{A}, i=1, \ldots, n$ according to some preference relation by taking into account criteria $c_{j} \in \mathrm{C}$. In this problem, it is mostly competition attitude which imposes itself. Selection of actions is not required. In this case, decision problem is formulated as follows: how rank competing candidates in decreasing order of merits including ties ?

So, ranking is assigning a rank position for each alternative in a subset $\mathrm{A}^{\prime}$ of $\mathrm{A}$; the same rank is assigned to alternatives whose data do not allow to decide between them [6], [7], [8]. Thus, to reach the ranking problem solution, classification process is required as shown in Fig. 2 from [9].

\section{Solving multicriteria ranking problem in group decision}

In literature, we found only one paper related to the subject: it is the adaptative fuzzy ELECTRE I method used in [10]. In this section, we present outline of the fuzzy ELECTRE I method and a new scalarizing function, the so-called CHEMATRE (Choix et Election des Meilleures Actions en Traduisant la REalité) for choosing and electing best alternatives expressing reality. Through this section we assume that data are geometric means so that one can completely rank competing alternatives.

\subsection{Fuzzy ELECTRE I method}

To solve multicriteria ranking problem in group decision, it is assumed that performance ratings are fuzzy numbers, especially trapezoidal one. Here is the outline of this method: 
- $\quad$ Each DM gives the weights for all criteria;

- $\quad$ Aggregate the fuzzy performance ratings with report to corresponding weights;

- $\quad$ Convert different criteria scales into comparable one via a linear transformation;

- $\quad$ Found the fuzzy decision matrix;

- $\quad$ Define both concordance and discordance matrix for each pairwise comparison of alternatives;

- Determine boolean matrix $\mathrm{B}$ and $\mathrm{H}$, respectively according to minimum level of concordance and discordance;

- $\quad$ Use Hamming distance to introduce dominance relation between each pair of alternatives;

- $\quad$ Conclude by a graphical representation of the binary relation $\left(>,>^{-1}, \approx\right.$, ?) as presented in Fig. 3 .

To achieve all these steps, one must have à high background in fuzzy sets and fuzzy logic theories. Nevertheless, it seems possible to solve the multi-Decision Maker multicriteria ranking problem simply. So we try to use some wellknown scalarizing functions as weighted sum or variant of Chebychev forms. In the following subsection, we present solely the one which gives consistent results.

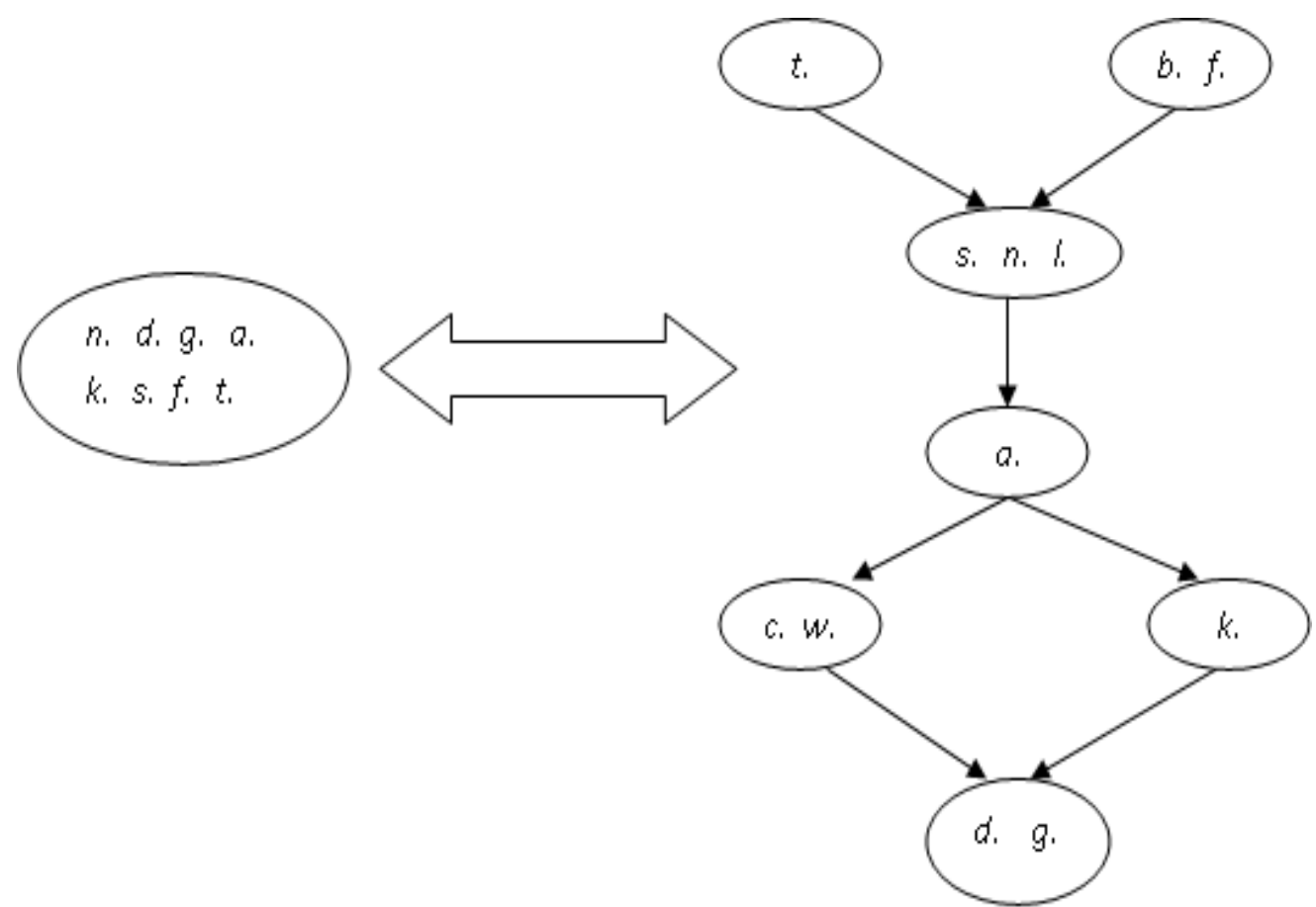

Fig. 2 : Ranking Problem Process
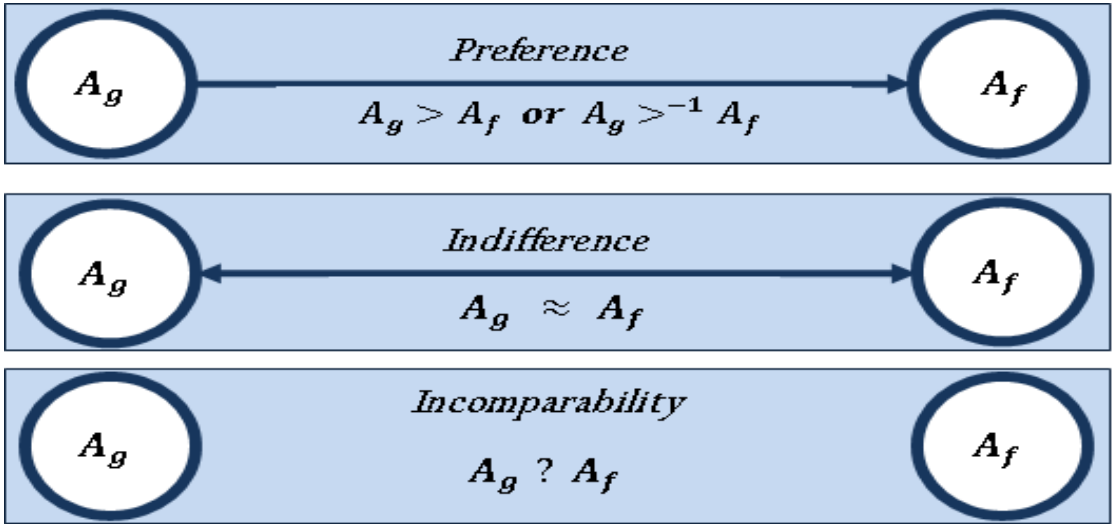

Fig. 3: Binary Relation Representation of ELECTRE I

\subsection{Chematre method}

This subsection is devoted to the so-called CHEMATRE method, the french for Choice and Election of Best Actions Expressing Reality. CHEMATRE belongs to methods whose principle are related to the Multi-Attribute Utility Theory (MAUT) [8]. So, in this subsection, we introduce notion on value function and aggregation of several additive value function. Before, let us define "geometric average" which is the value function here used. 


\subsubsection{Geometric average}

Definition 1: The geometric average of $\mathrm{n}$ values is obtained by the product of these $n$ values then taking the result $n^{\text {th }}$ root. Formally, let $r_{1}, r_{2}, \ldots, r_{n}$ be real numbers; their geometric average is

$$
M_{g}=\sqrt[n]{r_{1} \cdot r_{2} \cdot \ldots \cdot r_{n}}
$$

\subsubsection{Additive value function}

Let $G: A \subseteq \mathbb{R} \rightarrow \mathbb{R}$ à function such that

$$
G(a)=\sum_{j=1}^{m} \lambda_{j} v_{j}\left[g_{j}(a)\right]
$$

Where :

$\lambda_{j}$ is the weight of functiong $\mathrm{j}_{\mathrm{j}}, j=1, \ldots, n$;

$v_{j}\left[g_{j}(a)\right]$ is a monotonically increasing (respectively decreasing) function of $g_{j}$ to maximize (resp. minimize).

It is not restrictive to impose $0 \leq v_{j}\left[g_{j}\right] \leq 1$, and $\sum_{j=1}^{m} \lambda_{j}=1$ and that we deal with maximization problem. Then $G$ is an additive value function.

\subsubsection{Aggregation function of several additive value function models}

The general methodology for solving a multicriteria decision problem combines comparison operation and aggregation (or combination, fusion). The order in which these operations are made will determine two major types of approaches for multicriteria decision:

- " "aggregate then compare" (AC) approach: MAUT is the main representative of this category,

- "compare then aggregate" (CA) approach : outranking methods are representatives methods of this group.

ELECTRE I, II, IS, Tri are goods illustrations of that approach (see [11]). Clearly, CHEMATRE method uses "AC" strategy.

\subsubsection{Multi-attribute utility theory (MAUT)}

Let $E=E_{1} \times \ldots \times E_{r}, r \geq 2$, à set of objects where interests are described by a set $N:=1, \ldots, r$ attributes.

The objective of the multi-attribute utility theory is to numerically model preferences of a decision maker. These preferences are mathematically expressed as a binary relations that is represented by means of a global utility function $U: E \rightarrow \mathbb{R}$ Such that $a \geq b \Leftrightarrow U(a) \geq U(b), \forall a, b \in E$

It is usually assumed that the preference relation $\geq$ is complete and transitive (see eg. [12], In the case of non-transitive or incomplete preferences). Note that the most frequently used model for the overall utility function is additive utility model (see eg. [7], [14] and [15]).

The classical approach is based on the aggregation of decision criteria into a single criterion. It is about building a single synthesis criterion while using à scalarizing function $S$ by setting :

$$
g(a)=S\left(g_{1}(a), g_{2}(a), g_{3}(a), \ldots, g_{m}(a)\right) .
$$

In practice, the aggregation function $\mathrm{S}$ generally takes one of two forms:

1) Aggregation using weighted sum:

2) Additive aggregation :

$$
S\left(g_{1}(a), g_{2}(a), g_{3}(a), \ldots, g_{m}(a)\right)=\sum_{j=1}^{m} \lambda_{j} \cdot g_{j}(a) .
$$

\subsection{Outline of CHEMATRE method}

$$
S\left(g_{1}(a), g_{2}(a), g_{3}(a), \ldots, g_{m}(a)\right)=\sum_{j=1}^{m} \lambda_{j} v_{j}\left[g_{j}(a)\right] .
$$

Assume that $v_{j} \equiv 1_{\mathbb{R}}$ the identity function $\forall j=1, \ldots, m$.

- $\quad$ Define the matrix $w_{j}^{l}, l=1, \ldots, s$ and $j=1, \ldots, m$, criterion $j$ intra-weight for $l^{\text {th }} \mathrm{DM}$.

- $\quad$ Determine $G_{l}(a)=\sum_{j=1}^{m} w_{j}^{l} g_{j}^{l}(a), \quad \forall a \in A$

- $\quad$ Compute $U(a)=\sqrt[s]{\prod_{l=1}^{s} G_{l}(a)}, \forall a \in A$

- $\quad$ Operate pairwise comparisons: $\forall a, b \in A$, if $U(a) \geq U(b)$ then $a \geqslant b$ and if $U(a)=U(b)$ then $a=b$.

- $\quad$ Stop when all comparisons are made.

At the end, a complete or partial ranking is obtained in the set $A$ of alternatives. 


\subsection{Didactic examples}

In this section, we present two examples for performing CHEMATRE method. These examples were be solved by Rasmi Ginting [16].

\subsubsection{Example 1}

The problem is to find the best product between those in the set $A=\left\{P_{1}, P_{2}, P_{3}, P_{4}\right\}$, taking into account 5 criteria in $C=\left\{C_{1}, C_{2}, C_{3}, C_{4}, C_{5}\right\}$ where $C_{1} \equiv$ Production Award (unit: Franks / liter), $C_{2} \equiv$ Lifetime hinge (unit: years), $C_{3} \equiv$ Paint harmfulness (nominal scale: very little, Middle harmful, very harmful), $C_{4} \equiv$ Drying time, and $C_{5} \equiv$ Smell of paint (nominal scale: not strong, medium, high, very high). Data are provided by 3 DMs (or assigned to the criteria) in the form of notes. Rating scales extent may differ from one DM to another, and each one of criteria can be assigned a weighting coefficient. The result is a distribution on the set A of alternatives (products), one or more outperforming others.

The principle is the following: solution that outperforms others must be accepted by the largest possible number of DMs, and should not be rejected too much, even by one of them. Each DM builds his matrix of judgments with scales whose minimum value is 0 and maximum value is 10 .

For the Fuzzy ELECTRE I method used in [10], the winner is $P_{3}$.

Table 1: Judgment Matrix for DM 1

\begin{tabular}{|c|c|c|c|c|c|}
\hline & Price & Life & Odor & Drying & Harm \\
\hline Weight & 6 & 3 & 2 & 4 & 3 \\
\hline$P_{1}$ & 6 & 5 & 2 & 4 & 5 \\
\hline$P_{2}$ & 5 & 6 & 3 & 3 & 4 \\
\hline$P_{3}$ & 7 & 5 & 4 & 6 & 3 \\
\hline$P_{4}$ & 6 & 4 & 5 & 3 & 6 \\
\hline
\end{tabular}

Table 2: Judgment Matrix for DM 2

\begin{tabular}{|c|c|c|c|c|c|}
\hline & Price & Life & Odor & Drying & Harm \\
\hline Weight & 7 & 5 & 3 & 3 & 4 \\
\hline$P_{1}$ & 7 & 6 & 2 & 3 & 3 \\
\hline$P_{2}$ & 6 & 5 & 2 & 5 & 3 \\
\hline$P_{3}$ & 5 & 7 & 3 & 6 & 4 \\
\hline$P_{4}$ & 5 & 4 & 4 & 4 & 3 \\
\hline
\end{tabular}

Table 3: Judgment Matrix for DM 3

\begin{tabular}{|c|c|c|c|c|c|}
\hline & Price & Life & Odor & Drying & Harm \\
\hline Weight & 6 & 4 & 2 & 3 & 3 \\
\hline$P_{1}$ & 6 & 5 & 2 & 4 & 4 \\
\hline$P_{2}$ & 7 & 6 & 3 & 5 & 3 \\
\hline$P_{3}$ & 6 & 5 & 4 & 3 & 5 \\
\hline$P_{4}$ & 5 & 4 & 3 & 6 & 4 \\
\hline
\end{tabular}

CHEMATRE resolution.

Following Table 4 gives result of our methodology with the same set of weights where:

$G_{l}(P)=\sum_{j=1}^{5} w_{j}^{l} g_{j}^{l}(P)$

$U(P)=\sqrt[3]{\prod_{j=1}^{3} G_{l}(P)} \quad P \in\left\{P_{1}, P_{2}, P_{3}, P_{4}\right\}$

Table 4: Result of CHEMATRE

\begin{tabular}{|c|c|c|c|c|}
\hline$G_{l}(P)$ & $G_{1}(P)$ & $G_{2}(P)$ & $G_{3}(P)$ & $U(P)$ \\
\hline Product 1 & 86 & 106 & 84 & 91.48 \\
\hline Product 2 & 78 & 100 & 96 & 90.80 \\
\hline Product 3 & 98 & 113 & 88 & 99.14 \\
\hline Product 4 & 88 & 91 & 82 & 86.91 \\
\hline
\end{tabular}


Thus, overall alternatives scores are:

\begin{tabular}{cccc}
\multicolumn{5}{c}{ Table 5: Ranking of Products } & $P_{3}$ \\
\hline$P_{1}$ & $P_{2}$ & $P_{3}$ & 86.91 \\
II & 90.80 & 99.14 & IV \\
\hline
\end{tabular}

The above mentioned table 5 shows that $P_{3}$ is the best product. It is followed by $P_{1}$ and so on.

\subsubsection{Example 2}

The problem is to choose a partner from the following set: $A=\left\{\begin{array}{c}\text { Nippon Paint KK (P1), Courtaulds Coatings Holding (P2), Kansai Paint (P3), } \\ \text { International Paint (P4),US Sec of navy (P5) }\end{array}\right\}$

The set of criteria is:

$$
\mathrm{C}=\left\{\begin{array}{l}
\mathrm{C}_{1} \equiv \text { Product quality (nominal scale: Good, medium, Poor) } \\
\mathrm{C}_{2} \equiv \text { Technology (nominal scale : Good, medium, Poor) } \\
\mathrm{C}_{3} \equiv \text { Cost (unit : franks) } \\
\mathrm{C}_{4} \equiv \text { Time (unit : hour) }
\end{array}\right.
$$

A common preference scale to the four criteria was used. In practice this choice facilitates assignment of weights values associated to criteria. A partner with an average price is preferred. Indeed changing a preference scale that is associated to a criterion requires to change the weight value of this criterion in order to preserve the idea of compensation between criteria. Data are provided by DM (or assigned to the criteria by experts) as notes. The extent of rating scales may differ from one DM to another, and each criterion has a weighting factor. The result is a distribution on the alternatives set A, one or more alternatives that outperform others. Here is the principle: the alternative(s) which outperform(s) others must be accepted by a largest possible number of DMs, and should not be rejected too much, even by one of them. Each DM constructs its judgments matrix. Values scale lie from 0 to 10. For the Fuzzy ELECTRE I method used in [10], the winner is $\mathrm{P} 1$.

Table 6: Judgment Matrix for DM 1

\begin{tabular}{|c|c|c|c|c|}
\hline & $\mathrm{C}_{1}$ & $\mathrm{C}_{2}$ & $\mathrm{C}_{3}$ & $\mathrm{C}_{4}$ \\
\hline Weight & 4 & 3 & 2 & 5 \\
\hline P1 & 7 & 5 & 3 & 8 \\
\hline $\mathrm{P} 2$ & 3 & 6 & 8 & 4 \\
\hline P3 & 6 & 8 & 4 & 3 \\
\hline $\mathrm{P} 4$ & 5 & 4 & 6 & 7 \\
\hline P5 & 2 & 3 & 7 & 5 \\
\hline \multicolumn{5}{|c|}{ Table 8: Judgment Matrix for DM 3} \\
\hline & $\mathrm{C}_{1}$ & $\mathrm{C}_{2}$ & $\mathrm{C}_{3}$ & $\mathrm{C}_{4}$ \\
\hline Weight & 4 & 5 & 3 & 5 \\
\hline $\mathrm{P} 1$ & 8 & 3 & 6 & 7 \\
\hline $\mathrm{P} 2$ & 6 & 5 & 7 & 3 \\
\hline P3 & 5 & 8 & 4 & 2 \\
\hline $\mathrm{P} 4$ & 4 & 7 & 3 & 6 \\
\hline P5 & 7 & 6 & 5 & 8 \\
\hline
\end{tabular}

\begin{tabular}{ccccc}
\hline & $\mathrm{C}_{1}$ & $\mathrm{C}_{2}$ & $\mathrm{C}_{3}$ & $\mathrm{C}_{4}$ \\
\hline Weight & 3 & 4 & 3 & 5 \\
P1 & 6 & 8 & 9 & 4 \\
P2 & 4 & 5 & 6 & 7 \\
P3 & 7 & 6 & 8 & 4 \\
P4 & 6 & 8 & 4 & 7 \\
P5 & 5 & 4 & 7 & 6 \\
\hline
\end{tabular}

Table 7: Judgment Matrix for DM 2

CHEMATRE resolution.

Following Table 9 gives result of our methodology with the same set of weights: 
Table 9: Result of CHEMATRE

\begin{tabular}{ccccc}
\hline $\mathrm{G}_{1}(\mathrm{P})$ & $\mathrm{G}_{1}(\mathrm{P})$ & $\mathrm{G}_{2}(\mathrm{P})$ & $\mathrm{G}_{3}(\mathrm{P})$ & $\mathrm{U}(\mathrm{P})$ \\
\hline $\mathrm{P} 1$ & 97 & 89 & 100 & 95.21 \\
$\mathrm{P} 2$ & 85 & 66 & 85 & 78.12 \\
$\mathrm{P} 3$ & 89 & 71 & 82 & 80.31 \\
$\mathrm{P} 4$ & 97 & 79 & 90 & 88.35 \\
$\mathrm{P} 5$ & 82 & 56 & 113 & 80.35 \\
\hline
\end{tabular}

Where $G_{1}(P), U(P)$ with $P \in\{P 1, P 2, P 3, P 4\}$ are idem to those defined in equations (1) and (2) respectively. Thus, overall alternatives scores are:

Table 10: Ranking of Products

\begin{tabular}{ccccc}
\hline P1 & P2 & P3 & P4 & P5 \\
\hline 95.21 & 78.12 & 80.31 & 88.35 & 80.35 \\
I & V & IV & II & III \\
\hline
\end{tabular}

The above mentioned Table 10 shows that $\mathrm{P} 1$ is the best product. P4 is second-best and so on.

\section{Concluding remarks}

Thanks to interactive systems decision aiding, approaches to assist groups seeking a common solution have been developed.

Aggregation function presented in this paper combines concepts of geometric average and weighted sum average. Indeed, given the offsetting effect of the arithmetic average, method is desired resistant to manipulability by decision makers assigning high marks for important criteria to actions they wish to be selected. The geometric average then seems to be well indicated for this problem.

As PROMETHEE methods and all ELECTRE versions, CHEMATRE method is devoted to solve ranking problem. This is done with the usual real order on overall scores generated for each alternative, by the aggregating function which avoid compensation.

Results provided by CHEMATRE and those generated by the ELECTRE I method coincide for both examples presented here. Nevertheless CHEMATRE method does not use as long calculations as concordances and discordances matrices used in ELECTRE methods. So, for its simplicity and efficiency, CHEMATRE method is a representative method when dealing with ranking problem especially in presence of several DMs and criteria.

A challenge in this field is to pursue extensive numerical experiments, to compare results with other methods and to explore some extension of outranking such as ORESTE method for solving multi-Decision Maker multicriteria ranking problems.

\section{References}

[1] Denis Bouyssou, Thierry Marchant, Marc Pirlot, Alexis Tsoukiàs, and Philippe Vincke. Decision models with multiple criteria. Stepping stones for analyst. Springer, 2006.

[2] Sami Ben Mena. "Introduction aux méthodes multicritères d'aide à la décision", Biotechnol. Agron. Soc. Environ., 4(2) : 83 - 93, 2000.

[3] Abdelkader Adla. Aide à la facilitation pour une prise de décision collective : Proposition d'un modèle et d'un outil. PhD Thesis, Université de Toulouse, Juin 2010.

[4] Jean-Luc Marichal. Fonctions d'agrégation pour la décision. Brigham Young University, 2003.

[5] Slim Ben Khélifa. L'Aide Multicritère à la décision de groupe : L'approche du surclassement de synthèse. PhD Thesis, Université de Laval Québec, 1998.

[6] A. Bana Ecosta. "Les problématiques de l'aide à la décision : Vers l'enrichissement de la trilogie choix-tri-rangement", Revue Française d'automatique, d'informatique et de recherche opérationnelle, 30(2) : 191 - 216, 1996.

[7] Laurent Henriet. Système d'évaluation et de classification multicritère pour la décision. PhD Thesis, Université Paris-Dauphine, Janvier 2000.

[8] Bernard Roy. Méthodologie multicritère d'aide à la décision. Economica, Paris, 1985.

[9] Belacel Nabil. Méthodes de classification multicritère : Méthodologie et Applications à l'aide au diagnostic médical. PhD Thesis, Université Libre de Bruxelles, 2000.

[10] Rasmi Ginting and Henri Dou. L'approche multidécideur multicritère d'aide à la décision, Technical Report, 2000.

[11] Michel Grabisch. Une approche constructive de la décision multicritère. PhD Thesis, Université Paris I - Panthéon Sorbonne, Février 2004.

[12] Denis Bouyssou and Marc Pirlot. "Nontransitive decomposable conjoint measurement". Journal of Mathematical Psychology, 46(6): 677 703, 2002. http://dx.doi.org/10.1006/jmps.2002.1419.

[13] Denis Bouyssou and Marc Pirlot. "Additive difference models without additivity and substractivity". Journal of Mathematical Psychology, 48(4): 263 - 291, 2004. http://dx.doi.org/10.1016/j.jmp.2004.04.002.

[14] Ch. Gonzales. "Additive utilities when some components are solvable and others not", Journal of Mathematical Psychology, 40: 141 - 151, 1996. http://dx.doi.org/10.1006/jmps.1996.0013.

[15] Ch. Gonzales. Utililités additives : existence et construction. PhD Thesis, Université Pierre et Marie Curie, Paris, 1996.

[16] Rasmi Ginting. Intégration du système d'aide multicritère et du système d'intelligence économique dans l'ère concurrentielle. PhD Thesis, Université de Droit et des Sciences d'Aix-Marseille, Janvier 2000. 